\title{
Quality Improvement Project to Improve Timeliness Between Bronchodilator Treatments from Emergency Department to Medical Wards
}

\author{
Jennifer R Cockerham MBA, Gary R Lowe MEd RRT-NPS RPFT, Randy Willis MBA RRT-NPS, \\ Ryan M Stecks RRT-NPS, and Ariel Berlinski MD
}

\begin{abstract}
BACKGROUND: Quality improvement methodology was applied to study sporadic reports that patients with asthma were not given bronchodilator treatments or assessed within an appropriate time frame when they were admitted from the emergency department to the medical ward. The goal was to increase the number of patients who had an interval between emergency department assessment/bronchodilator treatment and medical ward assessment/treatment of < $120 \mathrm{~min}$. METHODS: A flow chart diagram, a fishbone diagram, data collection, intervention implementation, and data monitoring and analysis were used in this study. Data were collected on a pre-test of change cohort of 227 subjects with asthma from January 2013 to March 2014. A test of change adding a Q2H respiratory therapist assessment and as needed bronchodilator treatment order while the subject was in the emergency department was implemented during May of 2014. These data were compared with a post-test of change cohort of 278 subjects with asthma from May 2014 to July 2015. Data collection for both cohorts included the time from the last assessment/bronchodilator treatment in the emergency department to emergency department discharge, the time from emergency department discharge to assessment/treatment in the medical ward, and the sum of these 2 time periods. Mean times (minutes) were noted, and comparisons were made using 2 -tailed independent $t$ tests with significance set at $P<.05$. Mean monthly times were also compared in process control charts. RESULTS: There was a $124 \%$ increase noted in the percentage of subjects who received bronchodilator treatment within 120 min, a 53\% increase within $180 \mathrm{~min}$, and a $19 \%$ increase within $240 \mathrm{~min}$. The interval time between treatments decreased $21 \%$. CONCLUSIONS: Through quality improvement methodology, the group was able to significantly decrease the time between the last assessment/bronchodilator treatment in the emergency department and the first assessment/treatment in the medical ward for subjects with asthma. Moreover, improvement was seen in all studied parameters despite similar volumes in emergency department visits. Key words: quality improvement; asthma exacerbation; bronchodilator; respiratory assessment; pediatric asthma; emergency department. [Respir Care 2016;61(12):1573-1579. (C 2016 Daedalus Enterprises]
\end{abstract}

\section{Introduction}

The National Heart, Lung, and Blood Institute defines asthma as a chronic lung disease that inflames and narrows

\footnotetext{
Ms Cockerham, Mr Lowe, Mr Willis, Mr Stecks, and Dr Berlinski are affiliated with Respiratory Care Services, Arkansas Children's Hospital, Little Rock, Arkansas. Dr Berlinski is also affiliated with the Pulmonary Medicine Section, Department of Pediatrics, University of Arkansas for Medical Sciences, Little Rock, Arkansas.

Dr Berlinski has disclosed relationships with Vertex, AbbVie, Aptalis Pharma, Genentech, Janssen Research and Development, Gilead, Teva, Philips, Novartis, the National Institutes of Health, and the Therapeutic Development Network. The other authors have disclosed no conflicts of interest.
}

the airways (http://www.nhlbi.nih.gov/health/healthtopics/topics/asthma. Accessed July 28, 2015). According to the Centers for Disease Control and Prevention, asthma affects 22.6 million people in the United States, accounts

\footnotetext{
Mr Willis presented a version of this paper at AARC Congress 2014, held December 9-12, 2014, in Las Vegas, Nevada. Ms Cockerham presented a version of this paper as an Editors' Choice abstract at AARC Congress 2015, held November 6-10, 2015, in Tampa, Florida.
}

Correspondence: Jennifer Cockerham, Slot 303, 1 Children's Way, Little Rock, AR 72202. E-mail: CockerhamJR@archildrens.org.

DOI: $10.4187 /$ respcare.04581 
for 1.8 million emergency department visits per year, and results in 439,000 hospital admissions (http://www.cdc. gov/asthma/most_recent_data.htm. Accessed July 28, 2015). Patients presenting to the emergency department with an asthma exacerbation receive medical interventions, including inhaled bronchodilator treatment. If the patient does not improve, he/she is hospitalized. Arkansas Children's Hospital had 248 admissions to medical wards for asthma in 2014.

\section{See the Related Editorial on Page 1706}

In 1994, Arkansas Children's Hospital implemented respiratory therapist (RT)-driven asthma protocols. A core group of RTs assess the patient using a scoring system to dictate reassessments and bronchodilator treatment frequencies. When a patient with asthma is admitted to a medical ward, a licensed independent practitioner writes an order for an RT-driven asthma protocol. RT-driven protocols create a consistent and standardized practice of treating patients while maintaining safety and reducing costs. ${ }^{1}$ For the first $12 \mathrm{~h}$ after hospital admission on the asthma protocol, the patient is evaluated every $2 \mathrm{~h}$ and receives bronchodilator treatments every $4 \mathrm{~h}$ and as needed every $2 \mathrm{~h}$. Anecdotal evidence indicated that there were occasionally prolonged intervals of time between the last bronchodilator treatment provided in the emergency department and the first assessment or bronchodilator treatment given in the medical ward when a patient was admitted. A prolonged interval between assessments/bronchodilator treatments can lead to family perceptions of "abandonment" and potential escalation of care between the decision to admit and arrival to the ward.

In 2011, Arkansas Children's Hospital implemented an internal quality improvement training course, based on the "Model for Improvement,"2 called Improvement U. The program teaches quality improvement methodology utilizing the Plan-Do-Study-Act (PDSA) process. It also teaches that quality improvement is data-driven, requires appropriate interventions, and includes cyclic reassessment of the intervention. A quality improvement study was undertaken by Respiratory Care Services to increase the percentage of patients who waited $<120 \mathrm{~min}$ between their last assessment/bronchodilator treatment in the emergency department and the first assessment/treatment in the medical ward.

\section{Methods}

The University of Arkansas for Medical Sciences institutional review board approved a quality improvement project to improve the prolonged intervals between assessments or bronchodilator therapy for patients transitioning

\section{QUICK LOOK}

\section{Current knowledge}

Improvement in a health-care setting requires change, but not all change results in improvement. Quality improvement methodology is frequently used in healthcare settings to understand and measure improvement in processes.

\section{What this paper contributes to our knowledge}

It is important to take the time to thoroughly study and understand a process, identify key stakeholders to elicit buy-in, use data to drive the intervention, and monitor outcomes to measure success when applying quality improvement methodology to a problem. Significant improvements in multiple studied parameters were seen in reducing the time between the last assessment/bronchodilator treatment in the emergency department and the first assessment/treatment upon arrival to the medical ward by applying quality improvement methodology.

from the emergency department to the medical ward at Arkansas Children's Hospital. A flow chart (Fig. 1) and a fishbone diagram (Fig. 2) were created to improve the understanding of the processes for therapy initiation and patient transition before the intervention was established.

A retrospective chart review was conducted for all subjects age 18 months to $21 \mathrm{y}$ admitted from the emergency department to the medical wards who were placed on the asthma protocol between January 2013 and March 2014. Those who were admitted to the pediatric ICU or the intermediate care unit were excluded because they followed a different process. The following data were collected: age, sex, race, time of last emergency department bronchodilator treatment, and time of the first assessment/treatment upon arrival to the medical ward. The time interval between the latter 2 events was calculated in minutes. The percentages of subjects receiving treatment in $<120,<180$, and $<240$ min were calculated. Age was calculated as the difference between the date of admission and the birthdate and expressed to one decimal place. Demographics of the pre-test of change cohort can be seen in Table 1.

A process control chart was used to plot the monthly mean data (Fig. 3). A process control chart displays the data around the mean and $3 \sigma$ (equivalent to one $\mathrm{SD}$ ) above and below the mean, also known as the upper and lower control limits. The graph also includes internal zones that correspond to $1 \sigma$ and $2 \sigma$ above and below the mean. If analysis of the data did not reveal special cause variations, mean and control limits were anchored. The following criteria were used to define a special cause ${ }^{3}$ : (1) a data point fell outside the control limits; (2) 2 of 3 successive 


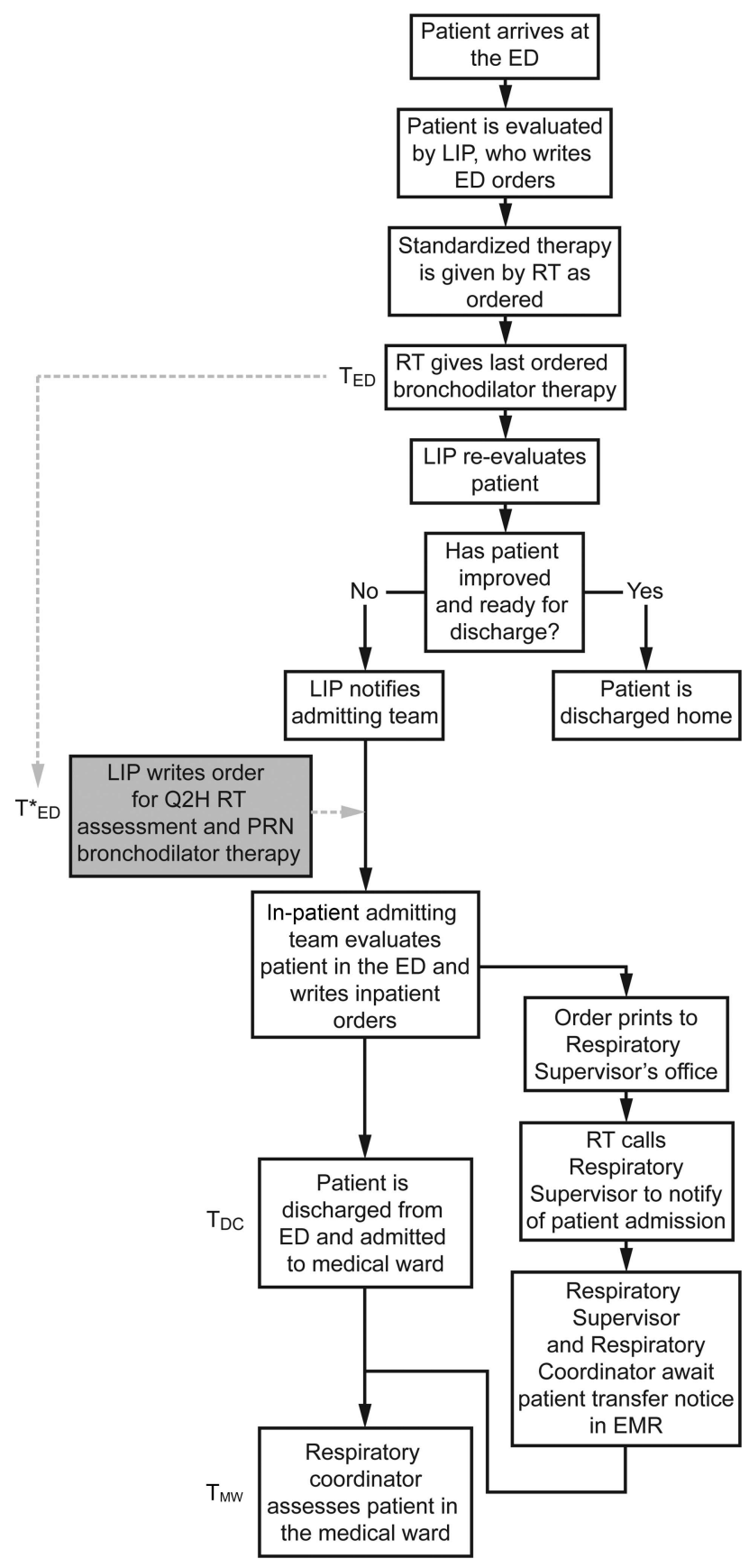

Fig. 1. The test of change goal was to shorten the time between the last respiratory therapist (RT) assessment/bronchodilator treatment given in the emergency department $\left(T_{E D}\right)$ and the first inpatient assessment/treatment $\left(T_{M W}\right)$. Before the test of change, $\mathrm{T}_{\mathrm{ED}}$ was the last bronchodilator treatment ordered and given before the decision to admit. With the intervention, $T_{E D}$ moves to $T^{\star}{ }_{E D}$ (still the time of the last emergency department RT intervention) and ensures that assessment for bronchodilator treatment continues to occur every $2 \mathrm{~h}$ after the in-patient licensed independent practitioner (LIP) writes the in-patient orders, even if there is a delay in admission. $T_{D C}$ is the recorded time of discharge from the emergency department and admission to the medical ward. ED = emergency department. points on the same side of the center line and $>2$ SDs from the center line; (3) presence of 8 points on the same side of the center line; (4) trends of $\geq 6$ sequential data points that increased or decreased. Review of the control chart showed that there were no special causes, and therefore the system was suited for an intervention.

Before implementation of an intervention, the flow chart and fishbone were re-analyzed. The interval between the last bronchodilator treatment in the emergency department and the first assessment/treatment in the medical ward was divided into 2 different periods. The first one included the time between the last bronchodilator treatment in the emergency department (see $\mathrm{T}_{\mathrm{ED}}$ in Fig. 1) and discharge from the emergency department (see $\mathrm{T}_{\mathrm{DC}}$ in Fig. 1). The second one occurred between the discharge from the emergency department $\left(\mathrm{T}_{\mathrm{DC}}\right)$ and the first assessment/treatment by a coordinator in the medical ward (see $\mathrm{T}_{\mathrm{MW}}$ in Fig. 1). To determine which part of the process to address, the emergency department discharge time (entered by the subject's nurse in the emergency department) was recorded, and the 2 time periods were calculated by subtracting the time of the last emergency department bronchodilator treatment from the emergency department discharge time and the emergency department discharge time from the time of the first assessment/treatment in the medical ward. The monthly means of the 2 time periods were placed into process control charts (Figs. 4 and 5), and there were no special causes, as determined by the outlined criteria. During this data collection period, admission and discharge time to the hospital were also recorded, and stay was calculated and recorded in minutes.

The monthly mean interval between emergency department and medical ward assessment/treatment was documented for 227 subjects in the pre-test of change cohort and showed a mean time of $202 \pm 26$ min. The percentages of subjects whose interval was $<120,<180$, and $<240 \mathrm{~min}$ are indicated in Table 1 .

The monthly mean interval between emergency department bronchodilator treatment and emergency department discharge was $147 \pm 26 \mathrm{~min}$, and the monthly mean interval between emergency department discharge and medical ward assessment/bronchodilator treatment was $54 \pm 9 \mathrm{~min}$, showing that the longest interval occurred when the subject was still in the emergency department waiting for admission.

Since the time from arrival to the medical ward until assessment/treatment by the coordinator was shorter, the team implemented an intervention that called for the emergency department licensed independent practitioner to write an order for a Q2H assessment and as needed bronchodilator treatment after the emergency department licensed independent practitioner made the decision to admit the subject. This kept the subject visible to the emergency department RT in the electronic medical record, ensured 


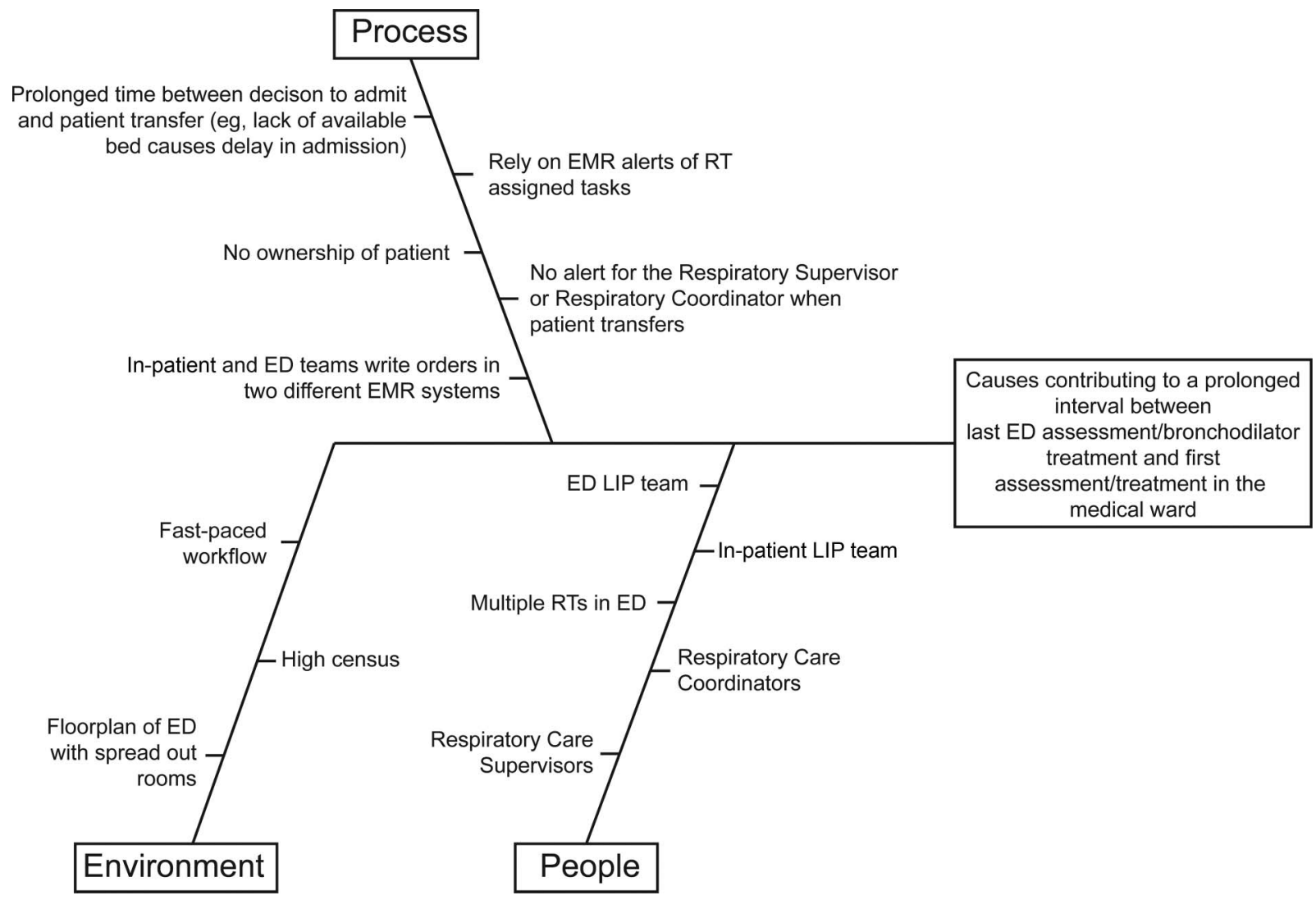

Fig. 2. Fishbone diagram outlining the causes contributing to a prolonged interval between last emergency department assessment/bronchodilator treatment and first in-patient assessment. EMR = electronic medical record, RT $=$ respiratory therapist, LIP = licensed independent practitioner, ED = emergency department.

Table 1. Demographics and Results of Pre- and Post-Test of Change Cohorts

\begin{tabular}{|c|c|c|c|}
\hline Characteristics & Pre-Test of Change & Post-Test of Change & $P$ \\
\hline Subjects, $n$ & 227 & 278 & NA \\
\hline Male sex, $n(\%)$ & $149(65.3)$ & $172(61.9)$ & .40 \\
\hline Age, mean \pm SD y & $6.6(4.1)$ & $5.8(3.7)$ & .02 \\
\hline African-American, $n(\%)$ & $141(62)$ & $181(65)$ & .52 \\
\hline Caucasian, $n(\%)$ & $67(21)$ & $78(28)$ & .77 \\
\hline Monthly percentage of subjects assessed within 120 min, mean \pm SD $\%$ & $17(10)$ & $38(15)$ & $<.001$ \\
\hline Monthly percentage of subjects assessed within 180 min, mean \pm SD $\%$ & $44(18)$ & $68(14)$ & .01 \\
\hline Monthly percentage of subjects assessed within 240 min, mean \pm SD $\%$ & $70(15)$ & $83(0)$ & $<.001$ \\
\hline Monthly time, mean $\pm \mathrm{SD}$ min & $202(26)$ & $160(24)$ & $<.001$ \\
\hline
\end{tabular}

that the emergency department RT continued to evaluate the subject, and moved $\mathrm{T}_{\mathrm{ED}}$ (representing the last $\mathrm{RT}$ intervention in the emergency department) closer to $\mathrm{T}_{\mathrm{MW}}$ in the process (see $\mathrm{T}_{\mathrm{ED}}$ in Fig. 1).

The next step in the quality improvement process was to create specific and measurable goals. During data analysis, it was determined that a small percentage of subjects had an interval of $<120$ min between the last bronchodilator treat- ment in the emergency department and assessment/treatment in the medical ward (Fig. 6). The aim was to increase the number of subjects who had an interval between emergency department assessment/bronchodilator treatment and medical ward assessment/treatment of $<120 \mathrm{~min}$ by $50 \%$ within 2 months of implementing a test of change. Secondary goals were to increase the percentage of subjects with an interval of $<180$ and $<240$ min for the amount of 


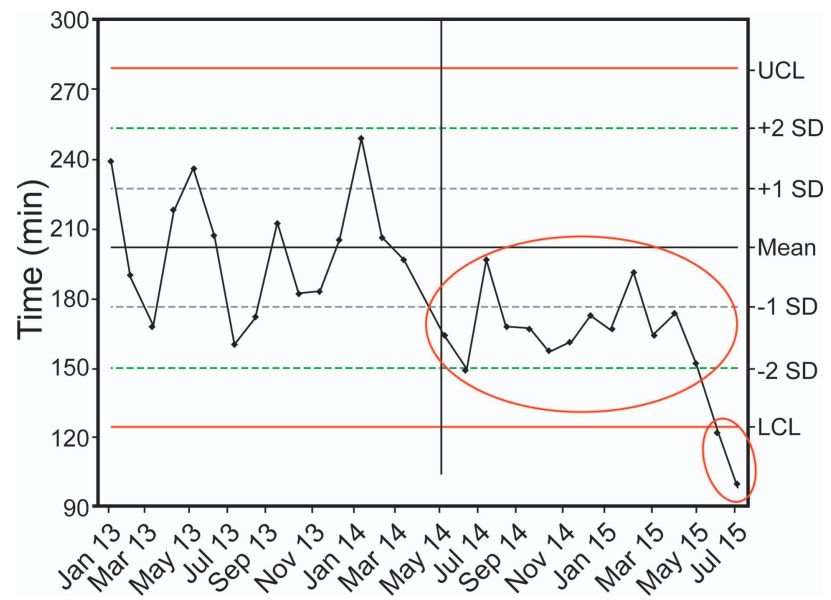

Fig. 3. Process control chart of the mean monthly time between emergency department assessment/bronchodilator treatment and in-patient assessment/treatment in the medical ward. Post-test of change the chart (shown by the vertical line) indicates a variance of special cause as noted by data points falling in the desired direction below the lower control limit and by the presence of $\geq 8$ points on the same side of the center line. The center horizontal line denotes the mean, and the upper and lower lines are the upper control limit (UCL) and lower control limit (LCL), respectively.

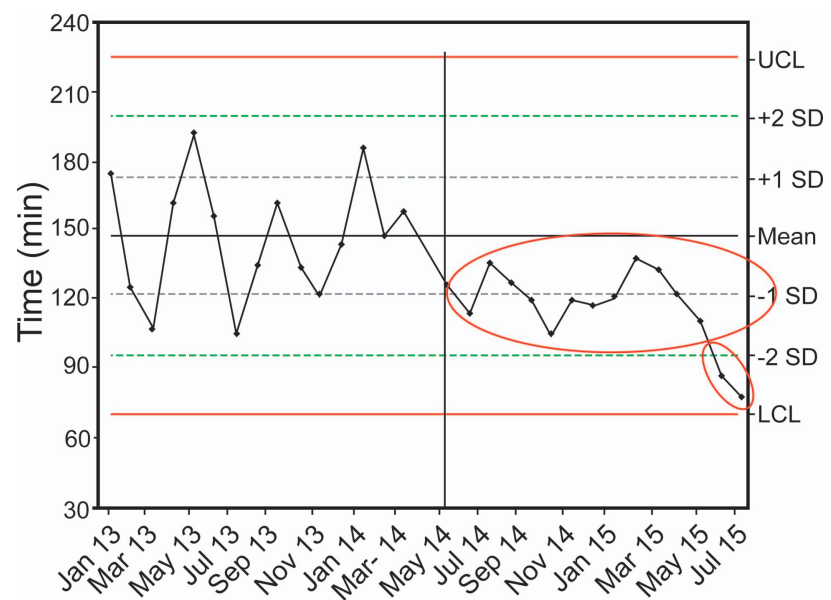

Fig. 4. Process control chart of the mean monthly time between emergency department assessment/bronchodilator treatment and emergency department discharge. Post-test of change (shown by the vertical line) on the chart indicates a variance of special cause as noted by presence of $\geq 8$ points on the same side of the center line and by the presence of 2 of 3 successive points on the same side of the center line and $>2$ SDs from the center line. The center horizontal line denotes the mean, and the upper and lower lines are the upper control limit (UCL) and lower control limit (LCL), respectively.

time between emergency department assessment/bronchodilator treatment and assessment/treatment in the medical ward and to decrease the overall time interval between treatments.

The test of change occurred in May 2014. Data were collected monthly on the post-test of change cohort with

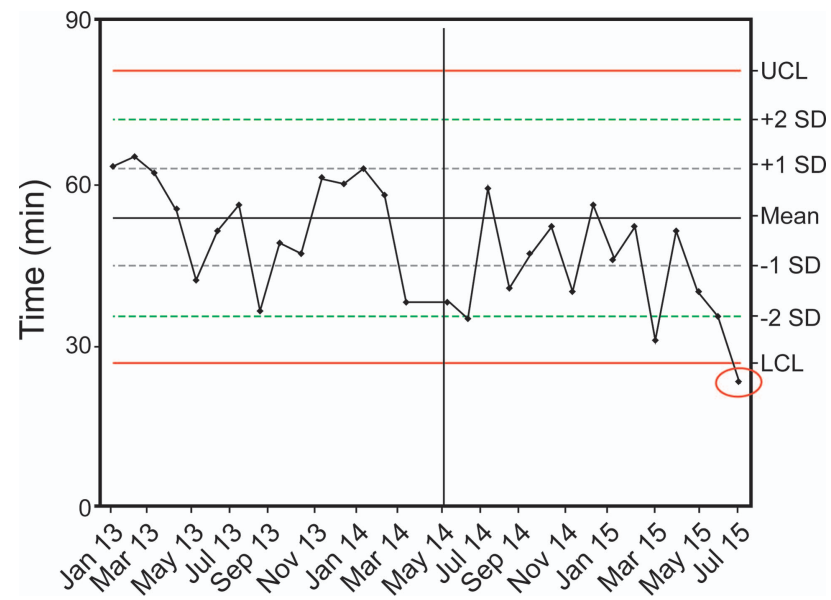

Fig. 5. Process control chart of the mean monthly time between emergency department discharge and assessment/treatment in the medical ward. Post-test of change (shown by the vertical line), the chart indicates a variance of special cause as noted by a data point falling in the desired direction below the lower control limit. The center horizontal line denotes the mean, and the upper and lower lines are the upper control limit (UCL) and lower control limit (LCL), respectively.

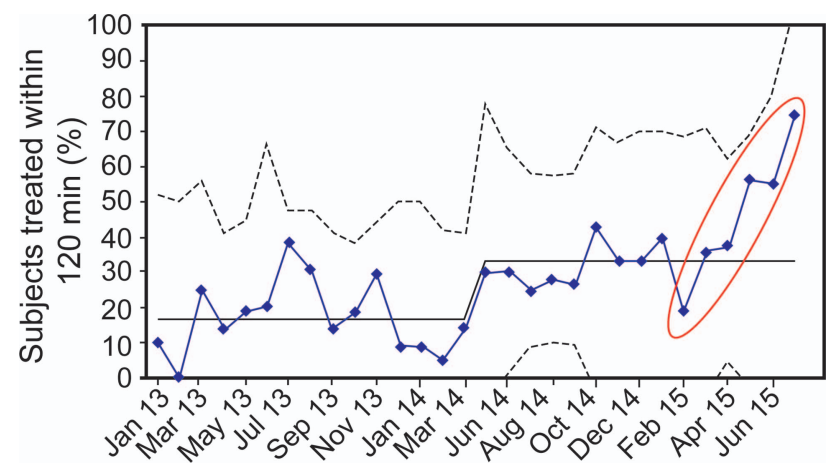

Fig. 6. Percentage of subjects treated within $120 \mathrm{~min}$. Post-test of change (shown by the vertical line), the chart indicates a variance of special cause noted by the presence of a trend of 6 sequential data points that increased. The center line denotes the mean, and dotted lines denote upper and lower control limits (UCL and LCL, respectively).

the same inclusion and exclusion criteria as the pre-test of change cohort from May 2014 through July 2015. There was a slight difference in the data collection for the posttest of change cohort in that the last assessment or bronchodilator treatment in the emergency department was recorded. The number of subjects seen in the emergency department during the study periods was extracted to rule out that any variation identified post-test of change could be attributed to lower emergency department volumes.

Continuous data comparing the monthly mean time interval between emergency department and medical ward assessments/treatments pre- and post-test of change were analyzed using 2-tailed independent $t$ tests of equal vari- 
ances with significance set at $P<.05$. The percentages of subjects treated within 120,180 , and 240 min were compared using a chi-squared test for independence and also using a $P$ chart. The latter was chosen because the denominator (total number of subjects included) varied every month. Special and common causes were determined using the criteria reported above. The percentage change of patients treated within 120, 180, and 240 min was calculated by subtracting the pre-test of change percentage from the post-test of change percentage, dividing by the pre-test of change percentage, and multiplying by 100 . The monthly mean times for the 3 time intervals were plotted in process control charts with the pre-test of change data. Emergency department volumes and stay data pre- and post-test of change were compared using 2-tailed independent $t$ tests of equal variances with significance set at $P<.05$.

\section{Results}

The interval between emergency department and medical ward assessment/bronchodilator treatment was documented for 278 subjects in the post-test of change cohort and showed a mean monthly time of $160 \pm 24 \mathrm{~min}$, which represents a $21 \%$ reduction when compared with baseline. The percentages of subjects whose interval was $<120$, $<180$, and $<240 \mathrm{~min}$ are indicated in Table 1. Both cohorts were similar in demographics, except the post-test of change cohort was 0.8 y younger $(P=.02$; see Table 1$)$. There was a $124 \%$ increase noted in the percentage of subjects who received an assessment/bronchodilator treatment within $120 \mathrm{~min}$, a $53 \%$ increase within $180 \mathrm{~min}$, and a 19\% increase within $240 \mathrm{~min}$. There was no difference in emergency department volumes pre- and post-test of change (pretest, 4,425 patients/month; post-test, 4,619 patients/month, $P=.19)$. Length of stay was also similar in both groups (pre-test, $799 \pm 347 \mathrm{~min}$; post-test, $826 \pm 348 \mathrm{~min}, P=.39$ ).

\section{Discussion}

Through the use of quality improvement methodology, the team was able to increase the number of children with asthma who had an interval of $<120$ min between the last assessment/bronchodilator treatment in the emergency department and the first assessment/treatment in the medical ward. The team was also able to decrease the actual time between assessments/treatments. The data are strong because they encompassed $100 \%$ of the potential cases, and the results were not affected by difference in emergency department volumes.

This quality improvement project provided several important lessons. For example, careful analysis of a process and gathering the right data related to that process were necessary before an appropriate intervention could be selected. This was performed by creating a detailed flow chart that depicted the entire process of the steps that occurred from patient arrival in the emergency department through assessment/treatment in the medical ward. Additionally, a fishbone diagram was constructed to determine which factors could be influenced by a test of change. Both of these tools proved invaluable in uncovering potential areas where changes could be implemented.

Quality improvement is a data-driven process, and this project described how well the quality improvement process worked, what happened when changes were applied, and documented successful performance. ${ }^{4}$ Without reliable data, there is no basis for determining whether there was a problem and where the problem occurred and no way to measure improvement after an intervention. Meticulous assessment of appropriately obtained data should direct the efforts to improve delivery of care. The team's hypothesis in the beginning was that the coordinators were the lag in the process. The team carefully evaluated the data and implemented an intervention before determining the specific variable affecting the prolonged intervals of delivery of medications. A large amount of data was collected before the implementation of the intervention to provide clarity on the magnitude of the problem. The anecdotal observations of prolonged intervals between treatments were validated during the pre-test of change data collection.

Quality improvement projects require continued monitoring to ensure that the results obtained through the improvement are sustained and to reduce the impact of drift, the gradual change in a process from the established, welldocumented way things should be done to the way things are actually done. ${ }^{4}$ Identifying the key stakeholders and fostering buy-in before implementation of a test of change were crucial to the success of the project.

The gains were sustained over time, as noted in Figures $3-6$, and data continue to be monitored. Future steps will include ways of optimizing the alerting of coordinators to patient arrival to the medical ward.

Although we improved the interval time between treatments, we were not able to demonstrate an effect on stay. We speculate that this could be due to the fact that stay is a multifactorial and complex variable. Discharge time is not a true representation of when a patient is clinically ready for discharge. If a patient is clinically ready for discharge at 2:00 AM, he/she would not realistically be discharged until the next day, thereby artificially increasing the stay. Social factors and care for other potential comorbidities might also extend stay. We speculate that the reduction in time resulting in more frequent assessments could have reduced the potential for parental perception of abandonment, but unfortunately, we did not collect these data. 


\section{Limitations}

First, there were no data available regarding the asthma severity and control classifications of each subject's chronic asthma. An emergency department asthma score also was not systematically recorded at the time of presentation (which would indicate severity of the exacerbation). ${ }^{5}$ These 2 measures would have given the team another comparator for patient population, thereby strengthening the argument that the intervention was the cause of the reduced intervals between the last emergency department assessment/bronchodilator treatment and the first assessment/treatment in the medical ward. Second, there could have been delays in documenting the assessment/treatment and discharge times that could result in variation between actual and documented times. Third, the generalizability of these changes could be limited due to difference in processes. However, the different steps that were taken in the quality improvement project should be easily applied in different settings.

\section{Conclusions}

Through quality improvement methodology, the group was able to significantly decrease the time between the last assessment/bronchodilator treatment in the emergency department and the first assessment/treatment in the medical ward for a patient with asthma. Moreover, improvement was seen in all studied parameters despite similar volumes in emergency department visits. It is important to take the time to thoroughly study and understand a process, identify key stakeholders to elicit buy-in, use data to drive the intervention, and monitor outcomes to measure success when applying quality improvement methodology to a problem.

\section{REFERENCES}

1. Metcalf AY, Stoller JK, Fry TD, Habermann M. Patterns and factors associated with respiratory care protocol use. Respir Care 2015; 60(5):636-643.

2. Langley GL, Moen RD, Nolan KM, Nolan TW, Norman, CL, Provost LP. The improvement guide, 2nd edition. San Francisco: JosseyBass; 2009:5.

3. Carey RG. Improving healthcare with control charts: basic and advanced SPC methods and case studies. Milwaukee: ASQ; 2003:16-17.

4. Smith L. Process drift: preventing the adulteration of management methods in clinical practices. J Med Pract Manage 2004;19(6):287-291.

5. National Heart, Lung, and Blood Institute. Guidelines for the Diagnosis and Management of Asthma. NIH Publication Number 085846. Bethesda, Maryland: National Institutes of Health; 2007.

This article is approved for Continuing Respiratory Care Education credit. For information and to obtain your CRCE

(free to AARC members) visit

www.rcjournal.com

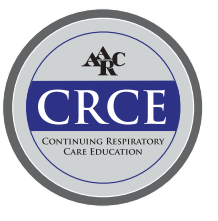

